\begin{tabular}{|c|l|}
\hline Title & Experimental study of cell pattern formation induced by internal heat sources in a horizontal fluid layer \\
\hline Author(s) & Takahashi, Jumpei; Tasaka, Y uji; Murai, Y uichi; Takeda, Y asushi; Y anagisawa, Takatoshi \\
\hline Citation & $\begin{array}{l}\text { International Journal of Heat and Mass Transfer, 53(7-8), 1483-1490 } \\
\text { https://doi.org/40.1016/.ijheatmasstransfer.2009.11.048 }\end{array}$ \\
\hline Issue Date & 2010-03 \\
\hline Doc URL & http://hdl.handle.net/2115/42808 \\
\hline Type & article (author version) \\
\hline File Information & IJHMT53.7-8_1483-1490.pdf \\
\hline
\end{tabular}

Instructions for use 


\title{
Experimental study of cell pattern formation induced by internal heat sources in a horizontal fluid layer
}

\author{
Jumpei Takahashi ${ }^{\mathrm{a}}$, Yuji Tasaka*a, ${ }^{*}$ Yuichi Murai ${ }^{\mathrm{a}}$, Yasushi Takeda ${ }^{\mathrm{a}}$, Takatoshi Yanagisawa ${ }^{\mathrm{b}}$ \\ ${ }^{a}$ Division of Mechanical Science, Graduate School of Engineering, Hokkaido University, Kita-13 Nishi-8, Sapporo \\ 060-8628, Japan \\ ${ }^{b}$ Institute for Research on Earth Evolution, Japan Agency for Marine-Earth Science and Technology (JAMSTEC), \\ Natsushima 2-15, Yokosuka 237-0061, Japan
}

\begin{abstract}
This study determines the flow structure in a convection cell with an internally heated layer by PIV to elucidate the convection cell transition mechanisms. The vertical velocity component is determined and the cell behaviour with respect to Rayleigh number is investigated quantitatively. Cell expansion process is described as a consequence of development of the descending flow at the centre of cells. The results suggest that a spoke-like structure is stable in this system in ideal conditions and a double-cell structure is formed when there are restrictions on the system, i.e. finite lateral boundaries.
\end{abstract}

Key words: Natural convection, Internal heating, Instability, Cell pattern formation, PIV

\section{Introduction}

Natural convection in a horizontal fluid layer is a basic phenomenon in heat transfer. Especially the so called Rayleigh-Bénard convection, which is due to external heating at the bottom of a fluid layer, is quite well known and appears in textbooks for heat transfer and fluid mechanics. Rayleigh-Bénard convection study has a long history since Bénard [1] and Rayleigh [2], and is still an important topic as a model of modal shift to turbulent flow [3, 4]. Parallel to these studies, natural convection induced by internal heat generation has been studied since the 1960s, and is also of great importance in the dynamics of the atmosphere of a planet [5] and in mantle convection in the earth [6,7], heat transfer relating to nuclear engineering [8], and other situations.

Convection cell structures with spatial periodicity in the flow pattern, are commonly seen in natural convection in shallow fluid layers. For example, when the fluid layer is heated from the bottom and cooled above, and the top surface is not enclosed (i.e. a Bénard-Marangoni convection), an orderly hexagonal cell structure is formed because of the effect of surface tension. Such hexagonal cells, Bénard cells, consist of an ascending flow region at the centre of the cell and a region with descending flow at the boundary to neighbouring cells. When the top surface is enclosed (i.e. a Rayleigh-Bénard convection), the flow pattern of convection cell strongly

\footnotetext{
${ }^{*}$ Corresponding author. Tel.: +8111706 6373; Fax.: +81 117067889

Email address: tasaka@eng.hokudai .ac.jp (Yuji Tasaka)

Preprint submitted to International Journal of Heat and Mass Transfer
}

March 24, 2010 
depends on the heating or cooling rates and the geometry of the boundary. A variety of patterns may be formed including two-dimensional rolls, concentric rings, hexagons and others, and there are many studies of such boundary-heating systems (ref. Koschmieder [9]).

In natural convection induced by internal heat generation of the fluid, the cell structure becomes hexagonal but the cell shape is greatly distorted and the flow direction is entirely opposite to a Bénard cell. The descending flow region appears at the centre and the ascending flow takes place at the periphery. For geophysical applications this kind of problem was first investigated experimentally by Tritton et al. [10], and theoretically by Roberts [11]. The investigations found large increases in cell scales in the horizontal direction with increases in Rayleigh number. Initially this increases was not thought to be an intrinsic feature of the problem but it was thought to be induced by characteristics of the experimental apparatus [12]. However, recent experimental studies $[13,14]$ and numerical simulation [15] has shown this to be an intrinsic feature of the problem.

The following cell transitions have further been reported $[13,14,15]$ : a descending flow region spreads to the periphery of the cell like the spokes of a wheel, termed a "Spoke-like structure" in the following; at higher Rayleigh numbers, additional cell structures are formed in the cell, namely an additional ascending flow appears at the centre surrounded by the descending flow, termed a "Double-cell structure" in the following. At still higher Rayleigh numbers, the hexagonal cell structure breaks down and a descending flow sheet becomes dominant $[15,16$, 17]. Fig. 1 is a schematic representation of the cell transitions in this system. However, the origin of these phenomena has not been elucidated; for example, the transitions in the cell structures do not agree with the earlier theoretical analysis and numerical simulation of an ideal condition even though the system appears simple. Therefore a quantitative experimental investigation of this phenomenon, e.g. measuring the temperature field or velocity field, is required to form the basis for a clarification of the reasons why convection cells undergo such a transition scheme. This paper attempts to investigate the cell transitions quantitatively in detail. Two-dimensional velocity vector fields are measured by Particle Image Velocimetry (PIV) for various Rayleigh numbers at different horizontal planes in the fluid layer. The distributions of the vertical velocity component are obtained from the vector fields by the numerical analysis and variations with Rayleigh number are investigated to determine the transitions.

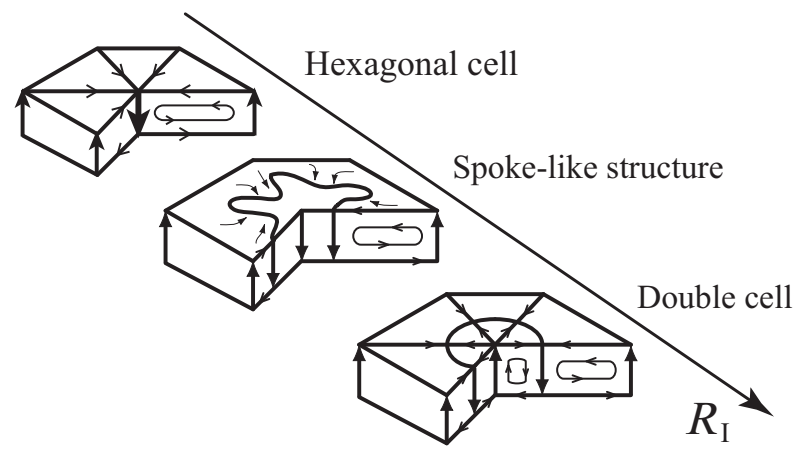

Figure 1: Schematic representation of cell transition; $R_{\mathrm{I}}$ is the internal Rayleigh number defined in eq. (1). 


\section{Experimental setup}

\subsection{Configuration}

The fluid layer configuration that is considered in this study is shown in Fig.2. The thickness of the fluid layer has been determined by a trade-off between the uniformity of internal heat generation (thicker fluid layer is better; the detailed explanation is in the end of this section) and a large aspect ratio for reducing the influence of the lateral boundaries (thinner one is better): Then the aspect ratio of the fluid layer is set to a sufficiently large value $(\Gamma=30)$. The size of the fluid layer is $210 \mathrm{~mm}$ square in the horizontal direction and $7 \mathrm{~mm}$ thick. To focus on the effect of internal heat generation, the thermal boundary conditions of the top and bottom are set to isothermal and adiabatic conditions, respectively.

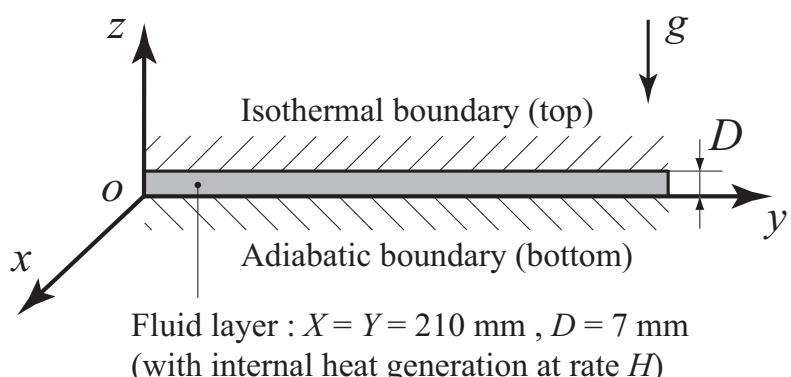

Figure 2: Configuration of the coordinate system, the thermal boundary conditions, and the size of the fluid layer.

The control parameter in this study is the internal Rayleigh number, which is defined as,

$$
R_{\mathrm{I}}=\frac{g \beta H D^{5}}{2 \lambda \kappa v}
$$

in an internal heating system, and where $D$ is the thickness of the fluid layer, $g$ is the gravity, $\beta$ is the thermal expansion coefficient, $\lambda$ is the thermal conductivity, $\kappa$ is the thermal diffusivity and $v$ is the kinematic viscosity of the fluid. The following sections use the reduced internal Rayleigh number, $R^{*}$, instead of $R_{\mathrm{I}}\left(R^{*}=R_{\mathrm{I}} / R_{\mathrm{c}}\right) ; R_{\mathrm{c}}$ is the critical Rayleigh number for the onset of convection, $R_{\mathrm{c}}=1386$, which was estimated theoretically by Roberts [11]. The critical value in the same apparatus we use here was verified experimentally [18].

The internal heat generation in the fluid layer is realised by Joule heating, which is generated by applying an electric current to the fluid layer at a rate $H$ per unit time and per unit volume. Alternating electric current is used to avoid bubble generation at the electrodes, and $H$ is derived as $H=E I / V$, where $E$ and $I$ are the effective values of the electric voltage and the current, and $V$ is the volume of the fluid layer. Preliminary experiments in a stationary condition confirmed that the voltage gradient in the fluid layer is constant parallel to the electrodes, and that the current between the electrodes is constant perpendicular to the electrode. Electric conductivity has slight temperature dependence and thus may cause non-uniformity in the temperature field even at the same horizontal plane. The study uses a thicker fluid layer than earlier studies $[10,12]$, and so non-uniformities in the heat generation are reduced; namely thicker fluid layers can make high $R_{\mathrm{I}}$ conditions without a non-uniformity in temperature field due to a high rate of the heat generation. 


\subsection{Experimental apparatus}

A schematic outline of the experimental apparatus for the configuration described above is shown in Fig.3. This apparatus is very similar to that used in previous work done by the authors $[13,14]$ with some parts improved for more ideal experimental conditions. The bottom plate of the fluid container consists of two glass layers separated by a vacuum to insulate from heat transfer. This plate is $6 \mathrm{~mm}$ thick including a $0.2 \mathrm{~mm}$ vacuum layer, it is transparent, allowing the fluid motion to be observed from the bottom through the plate. The top plate of the container, which cools the working fluid, is a copper plate. The thickness of the copper plate is $15 \mathrm{~mm}$ and it has rectangular channels for circulation of cooling water. Cooling water is circulated and maintained at a constant temperature, 20.0 degrees centigrade, by a thermostatic bath (HAAKE Phoenix II C35P, Thermo Electron Inc.). The channels sitting on the cooling plate and the lateral walls of the fluid layer are made of acrylic plate. As the internal heat generation is realised by applying electric current, the bottom surface of the copper plate has to be insulated electrically. A thin coating of black plastic paint is used for the electrical insulation and the paint layer would not interfere with the heat transfer between the working fluid and the copper plate as the thickness of the paint layer is $0.1 \mathrm{~mm}$ or thinner. The electric current is supplied from a stabilized AC power supply (EPX4104, NF Co.) and two rectangular graphite bars are mounted at the sides of the fluid layer as the electrode. Graphite is stable against electrolysis and no product generated due to electrolysis would invade the fluid layer. The terminals of the electric cables from the AC power supply were clamped on the electrodes by a screw. Potassium chloride was mixed in the water at 0.5 weight $\%$ to improve the electric conductivity of the water, as was also used in previous reports $[12,13,14]$ due to the weak temperature dependence of the electric conductivity. The electric conductivity of the fluid is $0.8 \mathrm{~S} / \mathrm{m}$ for 20.0 degrees centigrade; typical values of the electric current and voltage at $R^{*} \sim 8$ are $E=56.4 \mathrm{~V}, I=325 \mathrm{~mA}$ and then $H=57$ $\mathrm{kW} / \mathrm{m}^{3}$. The extent of temperature variation in the fluid layer is estimated by eq (2) and (3) [20]:

$$
\begin{gathered}
\Delta T=\frac{H D^{2}}{2 \lambda}, \\
\theta=5.95 R_{\mathrm{I}}^{-0.23} .
\end{gathered}
$$

The maximum of temperature difference in the conduction state, $\Delta T$, is calculated by eq (2) and the ratio of temperature difference between the conduction state and the convection state, $\theta$, is calculated by eq (3). At $R^{*} \sim 25\left(H=1.7 \times 10^{2} \mathrm{~kW} / \mathrm{m}^{3}\right)$, which is the maximum heat input, the temperature difference is estimated as about 4 degrees centigrade. 


\begin{tabular}{|lll}
\hline Nomenclature & & \\
$D$ & $:$ & thickness of the fluid layer \\
$E$ & $:$ & effective value of electric voltage \\
$g$ & $:$ & gravity \\
$H$ & $:$ & heat generation rate in the fluid layer \\
& & at per unit time and per unit volume \\
$I$ & $:$ & effective value of electric current \\
$r$ & $:$ & distance from the centre of cell \\
$R_{\mathrm{I}}$ & $:$ & internal Rayleigh number \\
$R_{\mathrm{c}}$ & $:$ & critical Rayleigh number \\
$R^{*}$ & $:$ & reduced internal Rayleigh number \\
$\boldsymbol{u}(u, v, w)$ & $:$ & velocity vector and its components \\
$U$ & $:$ & representative horizontal velocity \\
$V$ & $:$ & volume of the fluid layer \\
$w_{0}$ & $:$ & maximum descending velocity \\
$\widehat{w_{0}}$ & $:$ & of cold plume \\
& $:$ & maximum descending velocity \\
$w(r)$ & $:$ & obained in Gaussian fitting \\
$X, Y$ & $:$ & lateral length of the fluid layer \\
$z_{\text {lower }}, z_{\text {upper }}$ & $:$ & height of the illuminated plane \\
$\beta$ & $:$ & thermal expansion coefficient \\
$\Gamma$ & $:$ & aspect ratio of the fluid layer \\
$\Delta T$ & $:$ & maximum temperature difference \\
$\theta$ & $:$ & in conduction state \\
& $:$ & ratio of maximum temperature difference \\
$\kappa$ & $:$ & thetween conduction and convection state \\
$\lambda$ & $:$ & thermal conductivity \\
$\nu$ & $:$ & characteristic length of cold plume \\
$\sigma$ & & \\
& & \\
& &
\end{tabular}




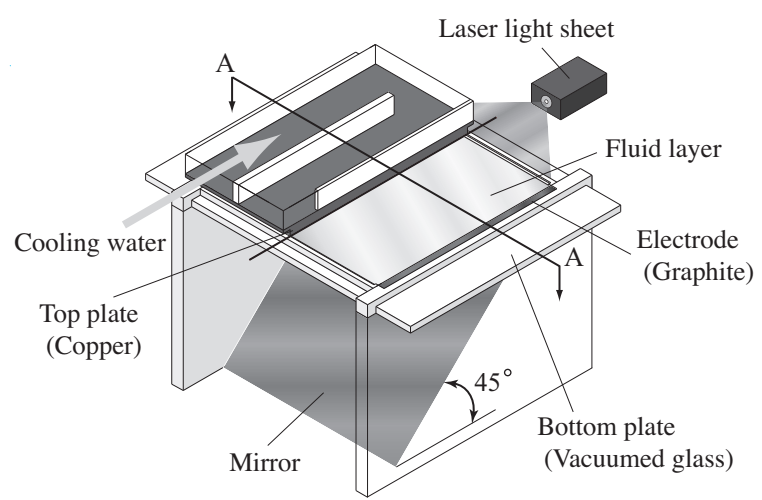

(a)

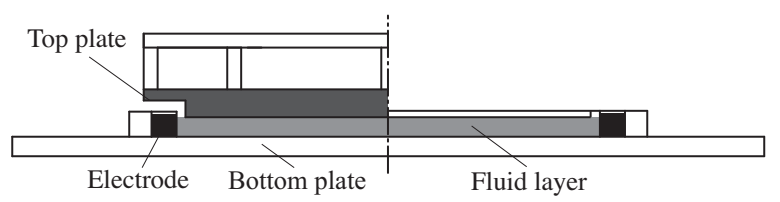

(b)

Figure 3: (a) Schematic outline of the experimental apparatus; the right-half of the figure shows the setup without the top plate and (b) A-A cross section of the fluid layer.

\subsection{PIV measurement and post processing}

Particle Image Velocimetry (PIV) is used to obtain the horizontal velocity distribution in the cells. Fine nylon particles (SP-500, Toray Industries Inc, specific gravity 1.02 and $5 \mu \mathrm{m}$ mean diameter) were used as tracer particles for the PIV measurements, and a green laser light sheet (wavelength : $532 \mathrm{~nm}$ ) was adopted for the illumination of a large area of the fluid layer in the horizontal direction. The laser light sheet illuminated a lower layer $\left(z_{\text {lower }}=2 \mathrm{~mm}\right)$ and an upper layer $\left(z_{\text {upper }}=5 \mathrm{~mm}\right)$ of the fluid layer, with the thickness of the illuminated area about $1 \mathrm{~mm}$. The sequence of the particle images were recorded by a digital camera (Nikon D300, 23.6 $\times 15.8$ mm CMOS, $3216 \times 2136$ pixels) at $1 \mathrm{fps}$ or $2 \mathrm{fps}$ rates, via the mirror mounted under the fluid layer at a 45 degrees tilt. Direct cross-correlation is used as the PIV algorithm.

Fig. 4(a) shows an original image of the particle distribution used in the PIV analysis. The particles appear to be uniformly dispersed in the fluid layer in spite of the very slow motion of the convection, like it is suitable for the PIV analysis. Fig. 4(b) is an instantaneous velocity distribution of the lower layer of the fluid layer. The white square in the top right corner indicates the size of $2 D$ for comparison with the cell size. The brightness of the grey scale arrows indicates the magnitude of the velocity. The spatial resolution and the velocity resolution of the velocity vector field are roughly $1.55 \mathrm{~mm}(\sim 0.22 D)$ and $0.05 \mathrm{~mm} / \mathrm{s}(\sim 2.33 \kappa / D)$ respectively. The velocity vector diverges at the centre of the cells, showing a descending flow at the centre of the cells, a typical feature of the convection cells appearing in an internally heated layer, and consistent with earlier studies [5, 12, 13, 14, 15].

Such a descending flow, a "cold plume" [19] in the following, is induced by a separation of the thermal boundary layer at the top plate in this configuration. The ascending flow at the periphery of the cells appears as a counter flow to this to satisfy mass conservation. The growth of the 


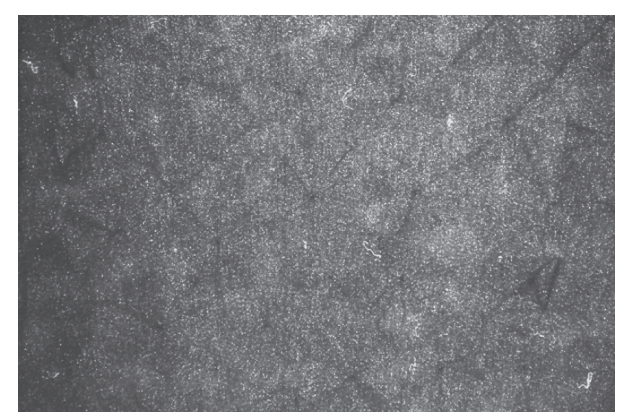

(a)

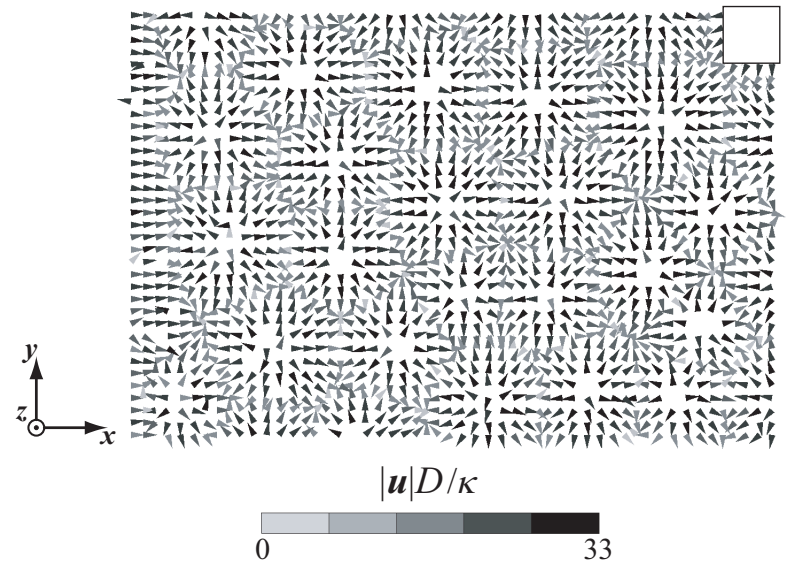

(b)

Figure 4: (a) Original image of the particle distribution and (b) instantaneous velocity distribution obtained at the lower layer $\left(R^{*} \sim 8\right)$; the white square indicates $2 D$ for comparison with the cell size; the brightness of the grey scale arrows indicate the magnitude of the horizontal velocity vector. 
cold plume with Rayleigh number, $R_{\mathrm{I}}$, makes the convective motion more active at the bottom part of the fluid layer, causing an increase in the horizontal size of the convection cell [13, 14]. This makes the magnitude of the descending velocity a suitable indicator to describe the flow transition in the cell, the vertical velocity component was calculated from the divergence of the two-dimensional velocity vector field obtained by PIV.

Characteristic time scales of the system are $X / U=420 \mathrm{sec}$ for global heat transfer in the fluid layer (where $X=210 \mathrm{~mm}$ is the horizontal scale of the fluid layer and $U=0.5 \mathrm{~mm} / \mathrm{s}$ is a representative magnitude of the horizontal velocity obtained in the experiment), and $D^{2} / \kappa$ $=327 \mathrm{sec}$ for local heat transfer in cells. Hence the measurement was started after about 2 hours from the initial stationary, isothermal condition of the fluid layer. The convection cells reach equilibrium state and the convective motion becomes quasi-steady, this makes it possible to determine a time-averaged velocity distribution reducing the large deviation in the vector field due to error vectors. Fig. 5(a) is an example of the time-averaged velocity vectors. In comparison with fig. 4(b), that is the instantaneous vector field, the averaged vector field appears sufficiently smooth to determine the spatial derivative. The vertical velocity component is estimated with the continuity equation for incompressible fluid,

$$
\frac{\partial u}{\partial x}+\frac{\partial v}{\partial y}+\frac{\partial w}{\partial z}=0
$$

as follows: From the time-averaged velocity distribution, the two-dimensional divergence in each grid point is calculated; and integrating the two-dimensional divergence over a vertical range gives the vertical velocity distribution as

$$
w(x, y)=-\int_{z_{1}}^{z_{2}} \nabla_{x y} \cdot \boldsymbol{u} d z, \quad \nabla_{x y}=\left(\frac{\partial}{\partial x}, \frac{\partial}{\partial y}, 0\right),
$$

where $\left(z_{1}, z_{2}\right)$ are set as $\left(0, z_{\text {lower }}\right)$ for the lower layer, and $\left(z_{\text {upper }}, D\right)$ for the upper layer. To reduce the noise, third-order fitting function for $\nabla_{x y} \cdot \boldsymbol{u}$ is calculated at each grid point on the horizontal plane from four values obtained at $z=0, z_{\text {lower }}, z_{\text {upper }}$ and $D$, and is used for the integration in eq. (5). Fig. 5(b) shows the contour lines of the calculated vertical velocity field. The vertical velocity, $w$, has the minimal values at the centre of the cells; increases rapidly around the centre and only gradually near the periphery of a cell.

\section{Results and discussions}

Fig. 6 shows vertical velocity distributions across a wide area of the fluid layer at three different internal Rayleigh numbers, (a) $R^{*} \sim 6$, (b) $R^{*} \sim 12$ and (c) $R^{*} \sim 20$. With increases in the internal Rayleigh number, the convective flow speed becomes faster and the horizontal scale of the cells becomes larger. The velocity of the cold plume at the centre of the cells is almost twice large as that of the ascending flow at the periphery of the cells. As shown in fig. 6(a) the shape of the convection cells is a quasi-regular hexagon and the arrangement of the cells appears regular, consistent with previous studies [10, 12, 13, 14, 15].

There are large changes in the shapes of the descending cold plume like there is in the shape of contour lines of the white and dark grey regions. The contour lines are circular at the relatively small $R_{\mathrm{I}}$ (fig. 6(a)); with increasing $R_{\mathrm{I}}$, the contours become distorted into a spoke-like shape as in fig. 6(b). This spoke-like structure was first reported in a report of numerical calculations [15] and the velocity distributions shown here are the first quantitative evidential experiment of this. 


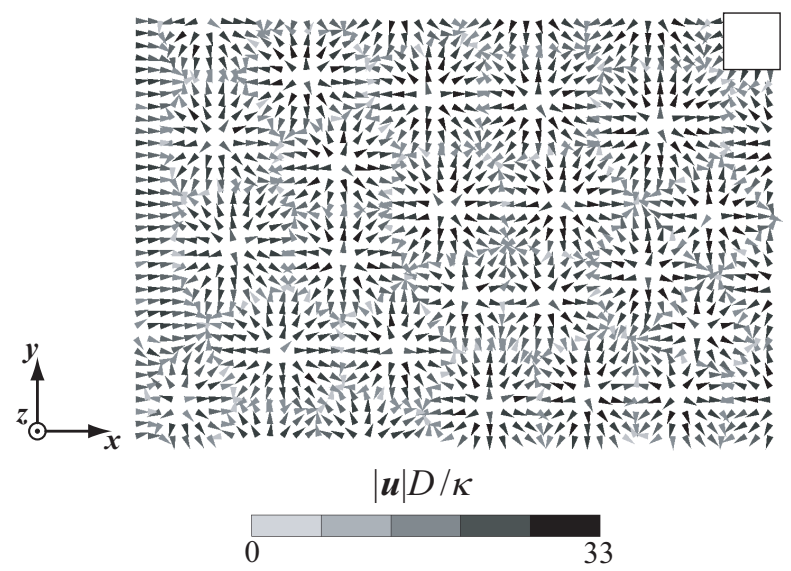

(a)

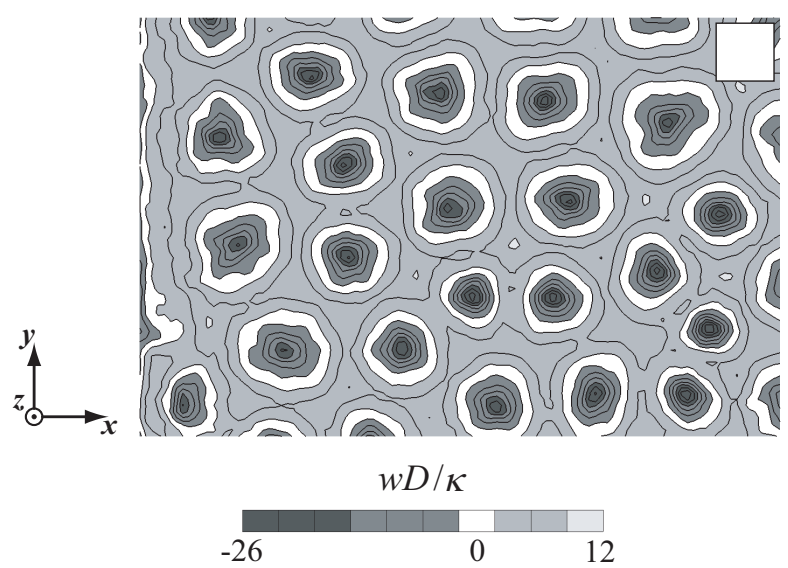

(b)

Figure 5: (a) Time-averaged horizontal velocity field, $(u, v)\left(|\boldsymbol{u}|=\left(u^{2}+v^{2}\right)^{1 / 2}\right)$, (b) contour lines of the time-averaged vertical velocity, $w$, at the lower layer $\left(R^{*} \sim 8\right)$; the white squares in the figures show $2 D$ for comparison with the cell size. 
Unlike the spoke-like structures appearing in the different natural convection systems [21, 22], the structure observed here is quite steady and has no local unsteadiness. Although the shape of cold plume (i.e. the descending flow region) has changed into the spoke-like shape, the boundaries of cells (i.e. the ascending flow region) and the position of the centre of cold plume do not change.

At relatively high $R_{\mathrm{I}}$, an additional ascending flow appears in some cells (fig. 6(c)): the new ascending flow divides a cold plume into two parts, and this is the onset of the transition to the double-cell structure reported in some experimental reports $[10,14]$. The centre of the cold plumes at which the descending velocity reaches the maximal is no longer at the centre of a cell, but such an irregular cell configuration is stable in the fluid layer in this condition. Observations of the transient state show that this structure is sometimes formed by a combination of two hexagonal cells. It occurs as a consequence of the cell size increase within the finite lateral boundaries, and such "double-cells" are formed by the effect of the lateral boundaries. Ichikawa et al. [15] supports this with their numerical simulation performed without lateral boundaries where only the spoke-like structure appears at a very similar $R_{\mathrm{I}}$. The restriction due to finite lateral boundaries of the fluid layer induces different types of cell size increases and flow instabilities in individual cells.

Due to the thermal boundary conditions at the top and the bottom of the fluid layer, and internal heat generation, the temperature profile in the stationary state has a parabolic shape in the depth direction of the fluid layer and it is not symmetrical to the vertical direction. Hence it may be expected that the convective motion is not symmetrical in the vertical direction; and numerical simulation has shown a different shape of an isothermal surface in the fluid layer. Fig. 7, 8, and 9 show details of the typical cell structure at the three values of $R^{*}$. Figures (a) and (b) show the horizontal velocity field, $(u, v)$, at the lower and upper layers, and (c) and (d) show the vertical velocity, $w$, distribution at the lower and upper layers. The H-shaped bars in the figures represent the relative scale of $2 D$. The structure of the cells in the depth direction are very similar although the temperature distribution is not linear in the depth direction as explained above. The horizontal velocity at the lower layer is faster than at the upper layer. Unlike the results of the numerical simulation, the "spokes" in the spoke-like structure are more distinct in the lower layer than in the upper layer as shown in fig. 8. This disagreement between the experimental and calculated results may be due to the imperfection of the adiabatic boundary at the bottom of the fluid layer or due to measurement errors: it is difficult to prevent a poor seed distribution in the upper part of the fluid layer. Overall, the results show that a high resolution in the depth direction is required to know details of the vertical structure of a cell.

For a more quantitative investigation on a statistical nature of the cold plume, Gaussian fitting of the velocity distribution in the descending flow region at the lower layer was performed. The Gaussian function is defined as

$$
w(r)=-\widehat{w}_{0} \exp \left(-\frac{r^{2}}{\sigma^{2}}\right),
$$

where $r$ is the distance from the centre of the cell, and $\widehat{w}_{0}$ and $\sigma$ are characteristic parameters for evaluating the velocity and the size of the descending flow region, respectively. The centre of the cell is determined by the following procedure: with the correlation between the Gaussian function and the over all obtained vertical velocity distribution, the position, where the correlation coefficient is the maximal, is considered the centre. Fig. 10 is an example of the fitting function $\left(R^{*} \sim 8\right)$ for the data points of the descending velocity in a one-dimensional expression. 


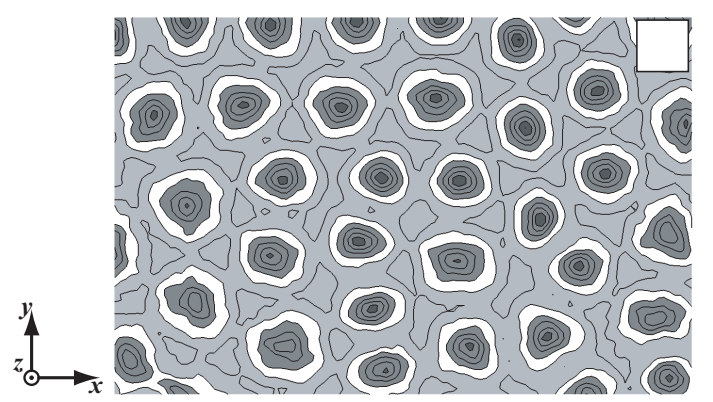

(a)

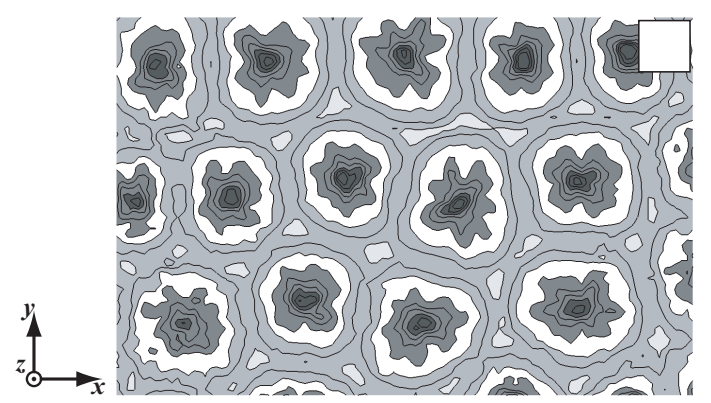

(b)

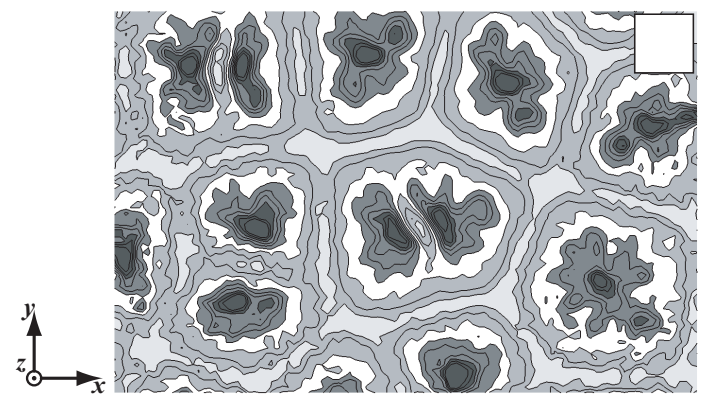

(c)

$w D / \kappa$

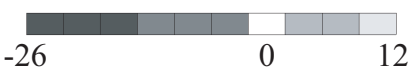

Figure 6: Vertical velocity distribution at the lower layer; (a) $R^{*} \sim 6$, (b) $R^{*} \sim 12$ and (c) $R^{*} \sim 20$; the shape of the cold plume is expressed by the contour lines between white and dark grey regions. 


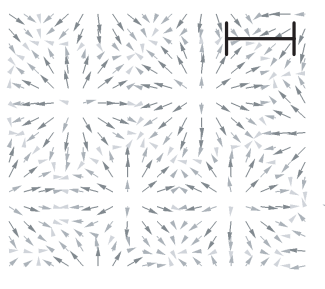

(a)

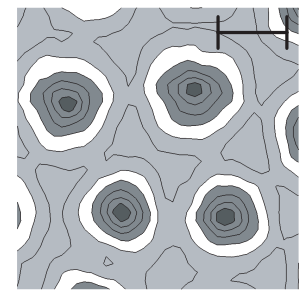

(c)

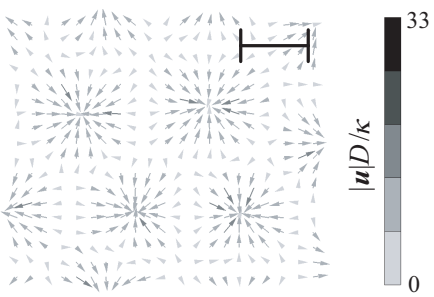

(b)

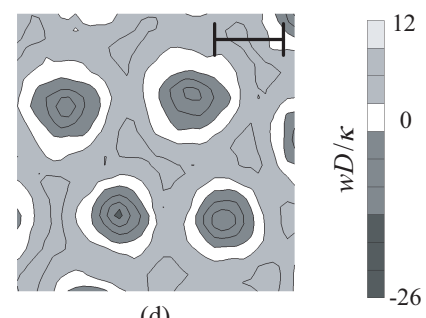

(d)

Figure 7: Two-dimensional velocity vector fields of $R^{*} \sim 6$ (a) the lower layer and (b) the upper layer: The corresponding vertical velocity distribution of (c) the lower layer and (d) the upper layer; H-shaped bars between the figures represent the scale of $2 D$.

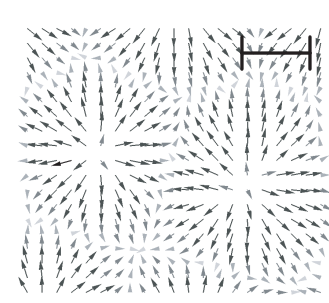

(a)

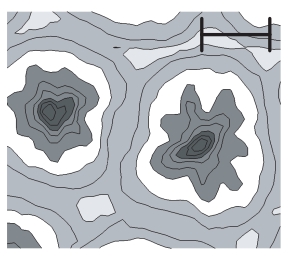

(c)

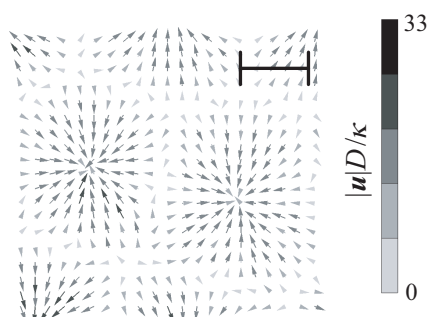

(b)

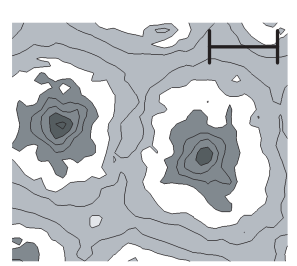

(d)

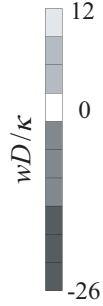

Figure 8: Two-dimensional velocity vector fields of $R^{*} \sim 12$ (a) the lower layer and (b) the upper layer: The corresponding vertical velocity distribution of (c) the lower layer and (d) the upper layer. 


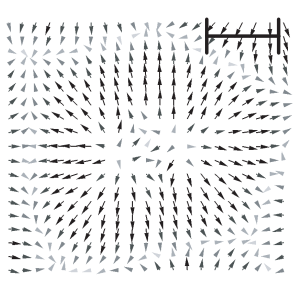

(a)

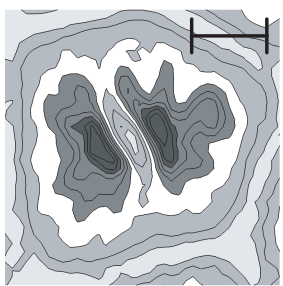

(c)

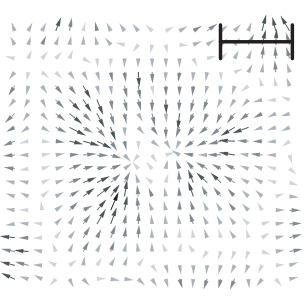

(b)

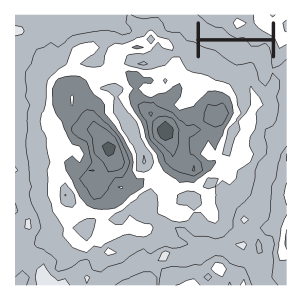

(d)
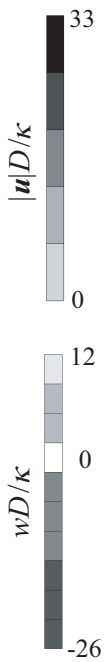

Figure 9: Two-dimensional velocity vector fields of $R^{*} \sim 20$ (a) the lower layer and (b) the upper layer: The corresponding vertical velocity distribution of (c) the lower layer and (d) the upper layer.

The plots of $w_{0}$ and $\sigma$ with respect to $R^{*}$ are shown in Fig. 11 (a) and fig. 11 (b) in doublelogarithmic representation; here $w_{0}$ is from the original vertical velocity at the centre determined by the Gaussian fitting, as $\widehat{w}_{0}$ usually underestimates the actual velocity at the centre of the cold plume (cf. fig. 10). Each data point in the graphs is an average of a number of cells (minimum 10 cells), and the error bars show the standard deviation.

These graphs show that the descending flow region becomes proportionally faster and wider as $R^{*}$ increases. It is also a quantitative confirmation of cell expansion with increasing the internal Rayleigh number $[10,12,13,14,15]$. From this it is possible to derive a power law for the variations in $w_{0}$ and $\sigma$ as

$$
\begin{gathered}
w_{0} D / \kappa=6.81 R^{* 0.51}, \\
\sigma / D=0.32 R^{* 0.42} .
\end{gathered}
$$

According to physical scaling on the thermal boundary layer formed at the top plate, the descending flow velocity, $w_{0}$ here, is proportional to the one-half power of $R^{*}$ [20], i.e. $w_{0} \sim R^{* 0.5}$. The exponent of the power law obtained in the experiment, 0.51 , approximately corresponds to the theoretically estimated exponent. At $R^{*}>20, w_{0}$ diverges from the power law and becomes smaller because the shape of the descending flow is modified by flow instability and is no longer a simple hexagonal shape. The variations in $\sigma$ still follow the power law for $R^{*}>20$ although the cell expansion has ceased in this region as reported in previous work [13]. The cell shape for $R^{*}>20$, however, has changed and cannot be modeled by a Gaussian distribution like at lower $R^{*}$. Like discussed on the power law about $w_{0}$, the second stage of instability occurs around $R^{*} \sim 20$, where the simple hexagon cell pattern changes into a double-cell structure. In comparison with the previous works $[13,14,15]$, the growth rate of the descending flow region in eq. (8) is close to the elongation rate of the cell; i.e. the cold plume is also elongate with respect to the cell dilatation in the same order until the transition occurs. 
Around $R^{*}=10$, at which the cell structure transfers from quasi-regular hexagon to spokelike structure in the observations, the variations of $w_{0}$ and $\sigma$ seem to be distinguishable to two regions. The discontinuous change may be induced by the transition to spoke-like structure, but it is difficult to be explained as a consequence of the transition because irregularities in the cell shape also increase. Further precision measurements of $w_{0}$ may give us the critical Rayleigh number for the onset of the spoke-like structure and relating discussions.

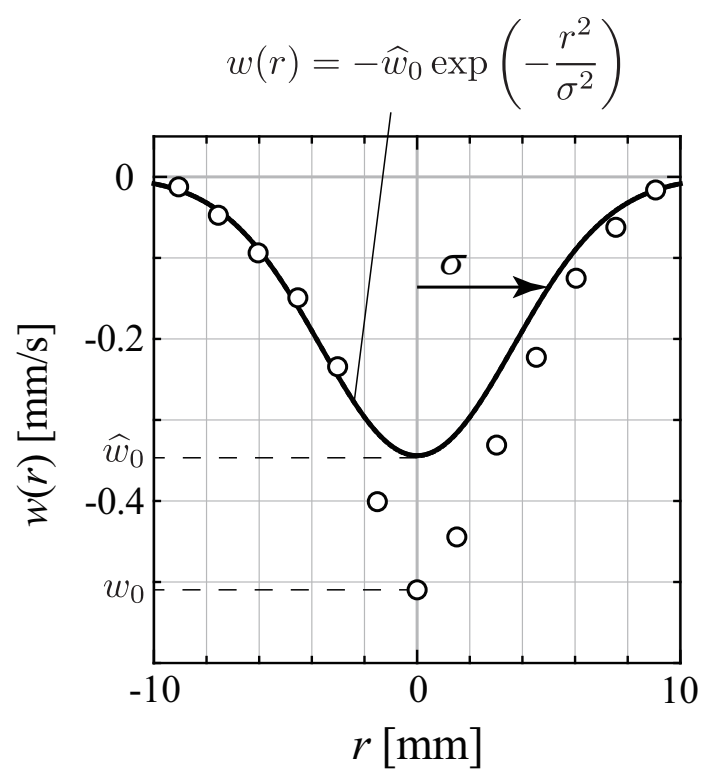

Figure 10: Gaussian fitting on data points of the descending velocity components in the cold plume (cf. eq. (4)) $\left(R^{*} \sim 8\right)$; here $w_{0}$ is the actual velocity at the centre of the cell determined by the fitting procedure.

\section{Conclusions}

Convection cell shape transitions induced by internal heat generation was studied experimentally. The horizontal and vertical velocity distributions at the upper part and the lower part of a fluid layer were obtained at various internal Rayleigh numbers, $R_{\mathrm{I}}$. Using PIV spoke-like structure was visualized quantitatively as contour lines of the velocity of the descending cold plume. Here spokes are observed as only a deformation of the descending cold plume, and there is no influence on the ascending flow component. Overall, the convection cell maintains its boundary shape as a quasi-regular hexagon. A double-cell structure in the velocity distribution, which has been observed in the numerical simulation previously, was also observed. The double-cell structure is formed by merging cells or the breaking up of cells due to the influence of the finite lateral boundary imposed in this study.

It was found that cell transition is strongly related to the development of the descending flow at the centre of the cell. Gaussian fitting was performed for a more quantitative investigation and the velocity of the descending flow was found to be consistent with the results of thermal boundary layer modeling up to $R^{*} \sim 20$. This suggests that at a higher Rayleigh number, the hexagonal cell structure changes into another stable state. In addition to this, a spoke-like structure was 


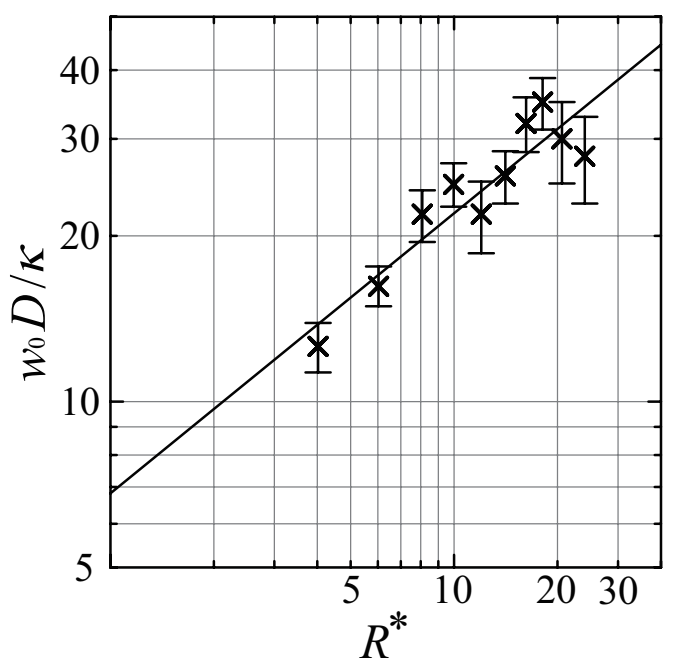

(a)

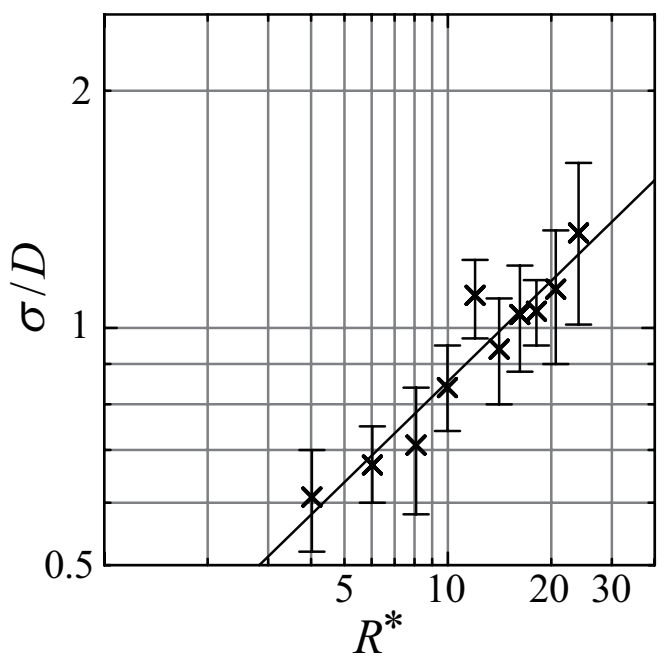

(b)

Figure 11: (a) The maximum descending velocity of the cold plume, $w_{0}$ versus the internal Rayleigh number, $R^{*}$; (b) characteristic length of the cold plume, $\sigma$ versus $R^{*}$ (double-logarithmic expressions). 
seen as the stable state in this system and the double-cell structure is formed by cells breaking up or combining. Cell combination occurs in the transient state for a quasi-steady state and is induced as a consequence of cell expansion within the finite lateral boundaries. For even higher Rayleigh numbers than those in this study, the spoke-like structure becomes a sheetlike structure $[15,16,17]$ for infinite horizontal fluid layers or finite fluid layers with only a small influence of the lateral boundary; the double-cell structure may become a two-dimensional roll structure as Schwiderski \& Schwab [12] suggested. Further works to establish this and to determine the critical Rayleigh number for the onset of the spoke-like structure are continuing and will be reported separately.

\section{References}

[1] H. Bénard, Les tourbillons cellulaires dans une nappe liquide, Rev. Gen Sciences Pure Appl 11 (1900) 1261-1271, 1309-1328

[2] Lord Rayleigh, On convection currents in a horizontal layer of fluid when the higher temperature is on the under side, Phil. Mag. 32 (1916) 529-546

[3] S. Newhouse, D. Ruelle, F. Takens, Occurance of strange axiom an attractors near quasi-periodic flows on $T^{m}$, $m \geq 3$, Commun. Math. Phys. 64 (1978) 35-40

[4] A. Libchaber, J. Manuer, Local probe in a Rayleigh-Bénard experiment in liquid helium, J. Phisigue Lett 39 (1978) L369-372

[5] D.J. Tritton, Internally heated convection in the atmosphere of Venus and in the laboratory Nature 257 (1975) $110-112$

[6] D.P. Mckenzie, J.M. Roberts, N.O. Weiss, Convection in the earth's mantle: toward a numerical simulation J. Fluid Mech. 62 (1974) 465-538

[7] S.A. Weinstein, P. Olson, Planforms in thermal convection with internal heat sources at large Rayleigh and Plandtl numbers, Geophys. Res. Lett. 17 (1990) 239-242

[8] T. Marimbordes, A. Ould E.L. Moctar and H. Peerhossaini, Active control of natural convection in a fluid layer with volume heat dissipation, Int. J. Heat \& Mass Trans. 45 (2002) 667-678

[9] E.L. Koschmieder, Bénard Cells and Taylor Vortices, Cambridge University Press, Cambridge, 1993

[10] D.J. Tritton, M.N. Zarraga, Convection in horizontal layers with internal heat generation: experiments, J. Fluid Mech. 30 (1967) 21-32

[11] P.H. Roberts, Convection in horizontal layers with internal heat generation: theory, J. Fluid Mech. 30 (1967) 33-49

[12] E.W. Schwiderski, H.J.A. Schwab, Convection experiments with electrolytically heated fluid layers, J. Fluid Mech. 48 (1971) 703-719

[13] Y. Tasaka, Y. Kudoh, Y. Takeda, T. Yanagisawa, Experimental investigation of natural convection induced by internal heat generation, J. Phys: Conference Series, 14 (2005), 168-179

[14] Y. Tasaka, K. Yonekura, Y. Takeda, T. Yanagisawa, Dilatation and pattern formation of cells in internally heated convection, J. Visualization 11 (2008) 213-220

[15] H. Ichikawa, K. Kurita, Y. Yamagishi, T. Yanagisawa, Cell pattern of thermal convection induced by internal heating, Phys. Fluids 18 (2006) 038101

[16] C.R. Carrigan, Multiple-Scale Convection in the Earth's Mantle: A Three-Dimensional Study, Science, 2154535(1982) 965-967

[17] C.R. Carrigan, Convection in an internally heated, high Prandtl number fluid: a laboratory study, Geophys. Astrophys. Fluid Dyn. 32 (1985) 1-21

[18] Y. Tasaka, Natural convection induced by internal heat generation, Ph. D. thesis, Hokkaido university (2005)

[19] R. Krishnamurti, Convection induced by selective absorption of radiation: A laboratory model of conditional instability, Dynamics of Atmos. and Oceans 27 (1997) 367-382

[20] D.L. Turcotte, J. Schubert, G. Schubert, Geodynamics 2nd edition, Cambridge Univ. press, Cambridge, 2001

[21] J.S. Szmyd, M. Jaszczur, H. Ozoe, Numerical analysis of the unsteady character of "spoke pattern" in Bridgman top seeding geometry, J. Crystal Growth 303 (2007) 241-245

[22] K.W. Yi, K. Kakimoto, M. Eguchi, M. Watanabe, T. Shyo, T. Hibiya, Spoke patterns on molten silicon in Czochralski system, J. Crystal Growth 144 (1994) 20-28 